\title{
Dimorphic Expression of Various Transcription Factor and Steroidogenic Enzyme Genes during Gonadal Ontogeny in the Air-Breathing Catfish, Clarias gariepinus
}

\author{
K. Raghuveer ${ }^{\mathrm{a}, \mathrm{c}} \quad$ B. Senthilkumaran ${ }^{\mathrm{a}} \quad$ C.C. Sudhakumari ${ }^{\mathrm{a}} \quad$ P. Sridevi ${ }^{\mathrm{a}}$
}

A. Rajakumar ${ }^{\mathrm{a}}$ R. Singh ${ }^{\mathrm{a}}$ R. Murugananthkumar ${ }^{\mathrm{a}} \quad$ K.C. Majumdar ${ }^{\mathrm{b}}$

a Department of Animal Sciences, School of Life Sciences-Centre for Advanced Studies, University of Hyderabad, and ${ }^{b}$ Centre for Cellular and Molecular Biology, Hyderabad, India; ${ }^{c} \mathrm{NICHD}$, National Institutes of Health, Bethesda, Md., USA

\section{Key Words}

Catfish · Dimorphic expression $\cdot$ Gonadal development •

Ovary $\cdot$ Sex differentiation $\cdot$ Teleosts $\cdot$ Testis

\begin{abstract}
In the present study the expression of 13 genes known to be involved in sex differentiation and steroidogenesis in catfish was analyzed during gonadal ontogeny by quantitative realtime RT-PCR. Dmrt1 and sox9a showed exclusive expression in male gonads while ovarian aromatase (cyp19a1) and fox 12 were abundant in differentiating female gonads. Most of the genes related to steroidogenesis were expressed only after gonadal differentiation. However, genes coding for $3 \beta$ hydroxysteroid dehydrogenase ( $3 \beta$-hsd), 17 $\alpha$-hydroxylase/ C17-20 lyase type 1 (cyp17) and steroidogenic acute regulatory protein (star) were barely detectable during gonadal differentiation. Ovarian aromatase, cyp19a1, which is responsible for estradiol-17 $\beta$ biosynthesis in females, was expressed very early in the undifferentiated gonads of catfish, around 30-40 days post hatch (dph). The steroidogenic enzyme, $11 \beta$-hydroxylase (cyp11b1) required for the production of 11 -ketotestosterone (11-KT) was expressed only after differentiation of testis. These results suggest that estradiol-17 $\beta$ has a critical role in ovarian differentiation, while the role of $11-\mathrm{KT}$ in testicular differentiation is doubtful. In conclusion,
\end{abstract}

dimorphic expression of $d m r t 1$ and sox9a in gonads during early development is required for testicular differentiation, and sex-specific expression of cyp19a1 and fox 12 in females plays a critical role in ovarian development. Our study reveals that the critical period of gonadal differentiation in catfish starts around 30-40 dph when sex-specific genes showed differential expression.

Copyright $\odot 2011$ S. Karger AG, Basel

Sexual reproduction is an important event which enables an organism to propagate and transfer genetic information from one generation to another. Successful reproduction usually depends on events that begin early in the reproductive life cycle. In vertebrates, sex determination and differentiation are the two important events in the development of gonads [Hughes, 2001]. Sex determination is the genetic (sex chromosomes) or environmental process by which the sex of an individual is established [Hughes, 2001; Kondo et al., 2009]. Sex determination is extremely diverse and lacks conservation in vertebrates. Sex differentiation refers to gonadal development after sex determination, when an indifferent/bipotential go-

This work was done at the University of Hyderabad.

\section{KARGER}

๑) 2011 S. Karger AG, Basel

Fax +4161306 1234

E-Mail karger@karger.ch

www.karger.com
Accessible online at:

www.karger.com/sxd
Prof. B. Senthilkumaran

Department of Animal Sciences, School of Life Sciences

University of Hyderabad, P.O. Central University

Hyderabad 500046, Andhra Pradesh (India)

Tel. +91 402313 4562, E-Mail bsksl@uohyd.ernet.in 
nad develops into testis or ovary, and is controlled by various factors/genes and hormones [Hughes, 2001]. Sex differentiation appears to be relatively conserved in vertebrates across the phyla. There are several factors like DMY/DMRT1, SOX9, AD4BP/SF-1 (NR5A1) and AMH which are implicated in testicular differentiation, while CYP19A1 and FOXL2 promote ovarian differentiation in most vertebrates [Hughes, 2001; Matsuda et al., 2002; Nanda et al., 2002; Nagahama, 2005; Guerrero-Estévez and Moreno-Mendoza, 2010]. Teleosts are excellent models for studying the events of sex determination and differentiation from an evolutionary point of view as they exhibit a broad range of sexual plasticity, ranging from hermaphroditism to gonochorism and from genetic to environmental sex determination [Devlin and Nagahama, 2002]. Further, their sex can be manipulated or completely reversed by exogenous sex steroid treatment around the critical period of sex determination/differentiation [Nagahama, 2005; Kobayashi et al., 2008; Raghuveer and Senthilkumaran, 2009].

Considering the knowledge gained in mammalian sex determination and differentiation studies, in-depth research work in teleosts related to this area is mostly restricted to daily or fortnight breeders like zebrafish, medaka and tilapia. In this regard, teleosts which show a seasonal (annual breeders) pattern of gonadal attenuation and recrudescence (reproductive cycle) are good models for comparative analysis and may provide interesting insights in understanding the expression pattern of sex-specific genes, not only during gonadal development, but also during gonadal recrudescence. Our laboratory is working on a teleost fish model, Clarias gariepinus (commonly known as North African/air-breathing catfish), in order to unravel the role and expression pattern of various transcription factors and steroidogenic enzyme genes during sex differentiation and gonadal development. The catfish is a gonochoristic annual breeder having a seasonal reproductive cycle. The present study combines the histological analysis of gonads and the expression pattern of genes/ factors during the process of gonadal ontogeny. We examined the sex specificity and precise timing of the expression of genes encoding transcription factors (wt1, dmrt1, sox $9 a$, sox $9 b$, sox 3 , ad $4 b p / s f-1$, foxl2), steroidogenic enzyme genes ( $3 \beta$-hsd, cyp17, 17 $\beta$ - $h s d 1, c y p 19 a 1, c y p 11 b 1)$ and the sterol transfer protein/steroidogenic acute regulatory protein coding gene (star) during gonadal differentiation and later stages of gonadal development after differentiation until maturity in catfish. For this purpose, the transcript levels of these 13 genes were analyzed by quantitative real-time PCR. Further, we also performed immu- nolocalization studies for few sex-specifically expressed proteins (Dmrt1, Sox9, Cyp19a1, and Foxl2) during gonadal development in catfish.

\section{Materials and Methods}

\section{Animals and Sampling}

Catfish (C. gariepinus) at different age groups were reared in fresh water tanks under ambient photothermal conditions. To obtain different age groups of catfish hatchlings, in vitro fertilization was performed during the breeding season using mature male and gravid female catfish injected (intraperitoneally) with human chorionic gonadotropin (500 IU/100 g body weight). The fertilized catfish embryos were transferred to small-size glass tanks containing filtered water with aeration. Generally catfish embryos take $24 \mathrm{~h}$ to hatch and the hatchlings can survive for 2-3 days without any external feed by utilizing the yolk sac for nutrition. Later on, the hatchlings were fed with live tubeworms until 3 months. Juvenile 3- to 8-month-old catfish were fed with commercially available fish feed pellets along with tubeworms. Catfish take about a year to mature which marks the beginning of the reproductive cycle. Catfish at different age groups $(0,10,20,30$, $40,50,75,100,150,200$ and 300 days post hatch (dph)) were collected and gonads were isolated for total RNA extraction as described earlier [Raghuveer and Senthilkumaran, 2010a]. In brief, the mesonephric gonadal complex (MGC) was isolated using fine sterile forceps under a stereoscopic dissection microscope from catfish hatchlings at 10,20,30 and $40 \mathrm{dph}$. The whole body was used for $0 \mathrm{dph}$ (less than $24 \mathrm{~h}$ after hatching). The MGCs of fishes of the same age group were pooled ( 5 hatchlings per sample; $\mathrm{n}=3$ ) to get a sufficient amount of total RNA. In catfish, morphological distinction of male and female gonads is possible from 50 dph onwards [Raghuveer and Senthilkumaran, 2009]. Therefore, male and female gonads of catfish ( 3 fishes per sample; $n=3$ ) at $50,75,100,150,200$ and $300 \mathrm{dph}$ were isolated using fine sterile forceps for total RNA extraction.

\section{Histological Studies}

The male and female gonads of catfish hatchlings at different age groups $(28,40,45,52,70,90$ and $160 \mathrm{dph})$ were fixed in Bouin's solution, dehydrated and embedded in paraplast (Sigma). For light microscopic histological examination, 5- $\mu \mathrm{m}$ sections were cut using a microtome (Leica, Wetzler, Germany) and stained with hematoxylin-eosin. All photomicrographs were taken with Olympus CX41 bright field light microscope (Olympus, Tokyo, Japan) fitted with a digital camera.

\section{Real-Time Quantitative RT-PCR ( $q R T-P C R)$}

All target genes analyzed and the endogenous control $(\beta$-actin) used in the present study were previously cloned from catfish. The GenBank accession numbers of the genes are as follows: Wilms' tumor gene 1, wt1 (JF510005); doublesex and mab-3 related transcription factor 1, dmrt1 (FJ596554); SRY-related homeobox gene 9, sox9a (HM149258) and sox9b (HM149259); SRY-related homeobox gene 3, sox3 (HQ680982); Adrenal 4 binding protein/steroidogenic factor 1, ad4bp/sf-1 (HQ680985); forkhead box L2, foxl2 (HQ680981); cytochrome P450 aromatase type A1 or ovarian aromatase, cyp19a1 (GU220075); 3ß-hydroxyster- 
Table 1. List of primers used for quantitative real-time PCR analysis

\begin{tabular}{lll}
\hline Gene & Forward primer $\left(5^{\prime} \rightarrow 3^{\prime}\right)$ & Reverse primer $\left(5^{\prime} \rightarrow 3^{\prime}\right)$ \\
\hline$w t 1$ & ACGCGCACAGGGTGTTCGA & GGTACGGTTTCTCTCCTTGTG \\
$d m r t 1$ & GCAGAGCTCAGCAAAACCCGG & GCGGCTCCCAGAGGCAGCAGGAGA \\
sox $9 a$ & TCTGGCGGCTGCTGAATGAAGG & CTCGGTATCCTCGGTTCACC \\
sox $9 b$ & GAGACCCAGTCAGGCCACAG & AGGGTCTCGATGTGGGCCA \\
sox3 & CACGGTATGAGTAGCCCACCA & GCGATGGCAGGTGGTGGTGAG \\
$a d 4 b p / s f-1$ & TCACTATG CACCTGCCT & CGCTTGTACATGGGGCCGAAC \\
foxl2 & CATGGCTATACGCGACAGCTC & CCAGTAGTTCCCCTTCCTCTC \\
$3 \beta-h s d$ & GAGGTAAATGTGAAAGGTACCAA & TAGTACACAGTGTCCTCATGG \\
$c y p 17$ & CCATGGCTCCAGCTCTTTCC & CAGTAAGACCAACATCCTGAGTGC \\
star & TCGTCCGAGCCGAGAACGG & TGCCTCCTCCACTCCACTG \\
$c y p 19 a 1$ & AGGTCCCTGGTTTTGTCTG & TGCAGATGGCCTGCTGAGG \\
$17 \beta-h s d 1$ & GACATCCTGGTGTGTAATGCAGG & CTGCCTGTGACCAGGATCCGT \\
$c y p 11 b 1$ & GGCAGTGGAGCGAATGCTGAA & GCACCCCGGGGAACCAGC \\
$\beta-a c t i n$ & ACCGGAGTCCATCACAATACCAGT & GAGCTGCGTGTTGCCCCTGAG \\
\hline
\end{tabular}

oid dehydrogenase $/ \Delta 4-\Delta 5$ isomerase, $3 \beta$-hsd (HQ680983); $17 \alpha$ hydroxylase/C17-20 lyase type 1, cyp17 (FJ790422); steroidogenic acute regulatory protein, star (FJ793811); 17 $\beta$-hydroxysteroid dehydrogenase type1, 17 $\beta$-hsd1 (HQ680984); and cytochrome P450 hydroxylase type B or 11 $\beta$-hydroxylase, cyp11b1 (HQ680986). Total RNA was extracted from gonadal samples of different stages using the Sigma TRI-reagent method and quantified using a NanoDrop spectrometer (NanoDrop Technologies, Wilmington, $\mathrm{DE}$ ). Reverse transcription (RT) was carried out with $1 \mu \mathrm{g}$ of total RNA and random hexamer primers using superscript-III (Invitrogen) according to the manufacturer's protocol. Successful RT was confirmed for all samples by performing PCR amplification of $\beta$ actin as an endogenous control. The primer sets used for qRT-PCR (table 1) were designed using Primer Express software (Applied Biosystems, Foster City, Calif., USA) such that at least one primer in each set flanked the intron-exon boundary to prevent amplification from genomic DNA. All qRT-PCRs were carried out in 20$\mu l$ reactions in triplicates using power SYBR Green PCR master mix (Applied Biosystems) in an ABI-7500 fast real time PCR machine (Applied Biosystems) at an initial hold of $95^{\circ} \mathrm{C}(10 \mathrm{~min})$ then followed by a 2 -step PCR reaction of $95^{\circ} \mathrm{C}(15 \mathrm{~s})$ and $60^{\circ} \mathrm{C}(1 \mathrm{~min})$ for 40 cycles according to the manufacturer's protocol. Dissociation or melting curve analysis was performed for each gene to check for single amplification. During PCR, fluorescence accumulation resulting from DNA amplification was recorded using the ABI 7500 sequence detection system (SDS) software (Applied Biosystems). Cycle threshold (Ct) values were obtained from the exponential phase of PCR amplification by SDS software. We used $\beta$-actin as an endogenous control as it did not show any significant change in expression at different stages. The target gene expression was normalized against $\beta$-actin expression generating a $\mathrm{Ct}$ value $(\mathrm{Ct}=\mathrm{Ct}$ of target gene $-\mathrm{Ct}$ of $\beta$-actin). Relative expression of the target gene was then calculated according to the equation $2^{-\Delta \mathrm{Ct}}$.

Immunohistochemistry

Immunohistochemistry (IHC) was performed to localize Dmrt1, Sox9, Cyp19a1 and Foxl2 proteins in differentiated gonads of catfish. For this, juvenile male and female catfish gonads at 52 dph were fixed in $4 \%$ paraformaldehyde in phosphate-buffered saline (PBS), processed and embedded in paraplast (Sigma). Sections of $5 \mu \mathrm{m}$ thickness were spread on sterile glass slides coated with polylysine, deparafinized in xylene and rehydrated in successively lower-graded concentrations of ethanol. The sections were then treated with $0.1 \% \mathrm{H}_{2} \mathrm{O}_{2}$ for 15 min to prevent endogenous peroxidase reaction. The sections were washed twice in $0.1 \mathrm{M}$ PBS, $1 \%$ Tween 20 and then blocked using 10\% normal goat serum (Bangalore Genei, Bengaluru, India) in $0.1 \mathrm{M}$ PBS for $10 \mathrm{~min}$ at room temperature $\left(25^{\circ} \mathrm{C}\right)$. Sections were then incubated overnight at $4^{\circ} \mathrm{C}$ either with Dmrt1, Sox9, Cyp19a1, or Foxl2 antibodies. The specificity of Dmrtl [Raghuveer and Senthilkumaran, 2009], Sox9 [Raghuveer and Senthilkumaran, 2010b] and Foxl2 [Sridevi and Senthilkumaran, unpublished data] antibodies used for IHC were evaluated previously in our laboratory. In brief, we used catfish-specific antibodies that were raised in our laboratory for the localization of Dmrt1 and Foxl2 proteins. For the detection of Cyp19a1, we used a heterologous antibody specific to tilapia Cyp19a1 protein. Human SOX9-specific antibody (heterologous) was used for detection of Sox9a in catfish [Raghuveer and Senthilkumaran, 2010b]. Following incubation with primary antibody, sections were washed in PBS, $0.1 \%$ Tween 20 (PBST) for $10 \mathrm{~min}$ and then incubated with 1:500 dilution of biotinylated anti-rabbit secondary antibody (Vector Laboratories, Burlingame, Calif., USA) for the horseradish peroxidase detection. Sections were incubated with $\mathrm{ABC}$ reagent (avidin-biotinylated horse-radish peroxidase complex) provided in VECTASTAIN Elite ABC kit (Vector Laboratories) for $30 \mathrm{~min}$ at room temperature. The sections were washed in PBS and color was developed using commercially supplied 3,3'-diaminobenzidine (DAB) as chromogen and $\mathrm{H}_{2} \mathrm{O}_{2}$ as substrate for horseradish peroxidase (Vector Laboratories). The sections were washed, dehydrated in graded ethanol series, cleared in xylene and mounted using DPX mountant. Immunofluorescence using FITC-conjugated anti-rabbit secondary antibody (Invitrogen) was employed for localizing Dmrtl protein. After the secondary incubation the sections were washed with PBS and mounted in $90 \%$ glycerol. Photomicrographs were taken using Olympus CX41 fluorescence cum light microscope. 
Statistical Analysis

The data from real-time PCR were expressed as mean \pm SEM of at least 3 independent samples. Significant differences in the data between male and female groups after differentiation ( $50 \mathrm{dph}$ onwards) were compared using Student's t test. A probability of $\mathrm{p}<0.05$ was considered statistically significant.

\section{Results}

Histological Observation of Gonadal Development in Catfish

Conventional histological methods were used to study the onset of gonadal sex differentiation in catfish. A primitive gonad with primordial germ cells (PGCs) surrounded by supporting cells was observed in the abdomen region near the coelomic cavity at $28 \mathrm{dph}$ (fig. 1A). At $40 \mathrm{dph}$, the formation of 2 bipotential gonads protruding from the mesonephros was observed (fig. 1B). Higher magnification of the bipotenial gonad showed development of germ cells surrounded by somatic/supporting cells (fig. 1C). The first sign of morphological differentiation of gonads was evident by formation of the ovarian cavity in the developing female gonad around $45 \mathrm{dph}$ (fig. 1D). The differentiated ovary at $52 \mathrm{dph}$ showed the presence of few meiotic oogonia and perinucleolar/primary growth oocytes (fig. 1E). The female gonads were completely filled with pre-vitellogenic oocytes and few perinucleolar oocytes at 70 and $90 \mathrm{dph}$ (fig. 1F, G) and showed different stages of oocytes (perinucleolar and pre-vitellogenic) at $160 \mathrm{dph}$ (fig. $1 \mathrm{H}$ ).

Testicular differentiation in catfish begins around 52 dph when developing germ cells (spermatogonia) surrounded by supporting/Sertoli cells were noticed (fig. 1I). Male and female gonads at 70 and 90 dph showed the proliferation and differentiation of spermatogonia and oocytes, respectively (fig. 1J, K). The completely developed testis at 160 dph showed different stages of spermatogonial cells, spermatocytes and few spermatids/sperm in the lumens indicating the progression of spermatogenesis (fig. 1L). These results suggest that testicular differentiation is delayed and takes more time than ovarian differentiation.

Expression Profile of Transcription Factors: wt1, $d m r t 1$, sox $9 a$, sox $9 b$, foxl2, ad $4 b p / s f-1$ and sox 3

In catfish, $w t 1$ expression was seen in the MGC before gonad differentiation, around 20-30 dph (fig. 2A). Its expression was found to be high in undifferentiated gonads at $40 \mathrm{dph}$. Later on, after differentiation of gonads ( $50 \mathrm{dph}$ onwards), wt1 was expressed at significantly higher levels in male gonads than in female gonads $\left({ }^{*} \mathrm{p}<0.05\right)$ (fig. $2 \mathrm{~A}$ ). However, its expression disappeared in both male and female gonads at maturity. The expression of $d m r t 1$ was observed at low levels in $40 \mathrm{dph}$ undifferentiated gonads (fig. 2B). Thereafter, its expression increased significantly in differentiated male gonads at $50 \mathrm{dph}$ and then gradually increased until $150 \mathrm{dph}$ (fig. 2B). On the other hand, $d m r t 1$ expression was negligible or undetectable in female gonads during gonadal ontogeny. Previously, we isolated 2 duplicate isoforms of sox 9 which were named sox $9 a$ and sox $9 b$ [Raghuveer and Senthilkumaran, 2010b]. In this study, sox $9 a$ and sox $9 b$ transcripts were first noticed at low levels in indifferent gonads at $30 \mathrm{dph}$ and at moderately high levels in bipotential gonads around 40 dph (fig. 2C, D). Later on, sox9a expression was observed only in male gonads at $50 \mathrm{dph}$ and gradually decreased until $100 \mathrm{dph}$ (fig. 2C). Then its expression remained stable in male gonads till maturity. Conversely, sox $9 \mathrm{~b}$ mRNA was detected at significantly high levels in female gonads at 50 and $75 \mathrm{dph}$. Thereafter, its expression decreased gradually marking the progression of ovarian development (fig. 2D). In developing male gonads, sox $9 b$ expression was barely detectable or negligible (fig. 2D). Expression of sox 3 was noticed from $0 \mathrm{dph}$ till $300 \mathrm{dph}$ (fig. 3A). It was almost similar in both male and female gonads from $50 \mathrm{dph}$ to $150 \mathrm{dph}$. However, its expression was significantly higher in males compared to females at 200300 dph ( $\left.{ }^{*} \mathrm{p}<0.05\right)$ (fig. 3A). Real-time PCR analysis revealed low levels of foxl 2 transcripts in the MGC of hatchlings at 20 and $30 \mathrm{dph}$ (fig. 3B). The transcript was present in undifferentiated gonads at $40 \mathrm{dph}$, increased considerably in female gonads at $50 \mathrm{dph}$ and thereafter decreased gradually till $100 \mathrm{dph}$ (fig. 3B). In male gonads, foxl2 was expressed at barely detectable or negligible levels throughout gonadal development $\left({ }^{* *} \mathrm{p}<0.001\right)$ (fig. 3B). In catfish, prominent expression of ad $4 \mathrm{bp} / \mathrm{sf}-1$ was seen in indifferent gonads at 30 and $40 \mathrm{dph}$ (fig. 3C). Thereafter, it is expressed in both differentiated male and female gonads until maturity, with significantly higher levels in females $\left({ }^{*} \mathrm{p}<0.05\right)$ (fig. 3C).

\section{Expression Profile of Genes Involved in}

Steroidogenesis: $3 \beta$-hsd, cyp17, star, cyp19al,

$17 \beta$-hsd 1 and cyp11b1

$3 \beta$-hsd, cyp17 and star transcripts were detectable at very low levels in undifferentiated gonads at $40 \mathrm{dph}$ (fig. 4A-C). These genes showed significantly higher expression in male gonads compared to female gonads during ontogeny $\left({ }^{*} \mathrm{p}<0.05 ;{ }^{* *} \mathrm{p}<0.001\right)$ (fig. $\left.4 \mathrm{~A}-\mathrm{C}\right)$. Further, their expression gradually increased in both gonads 

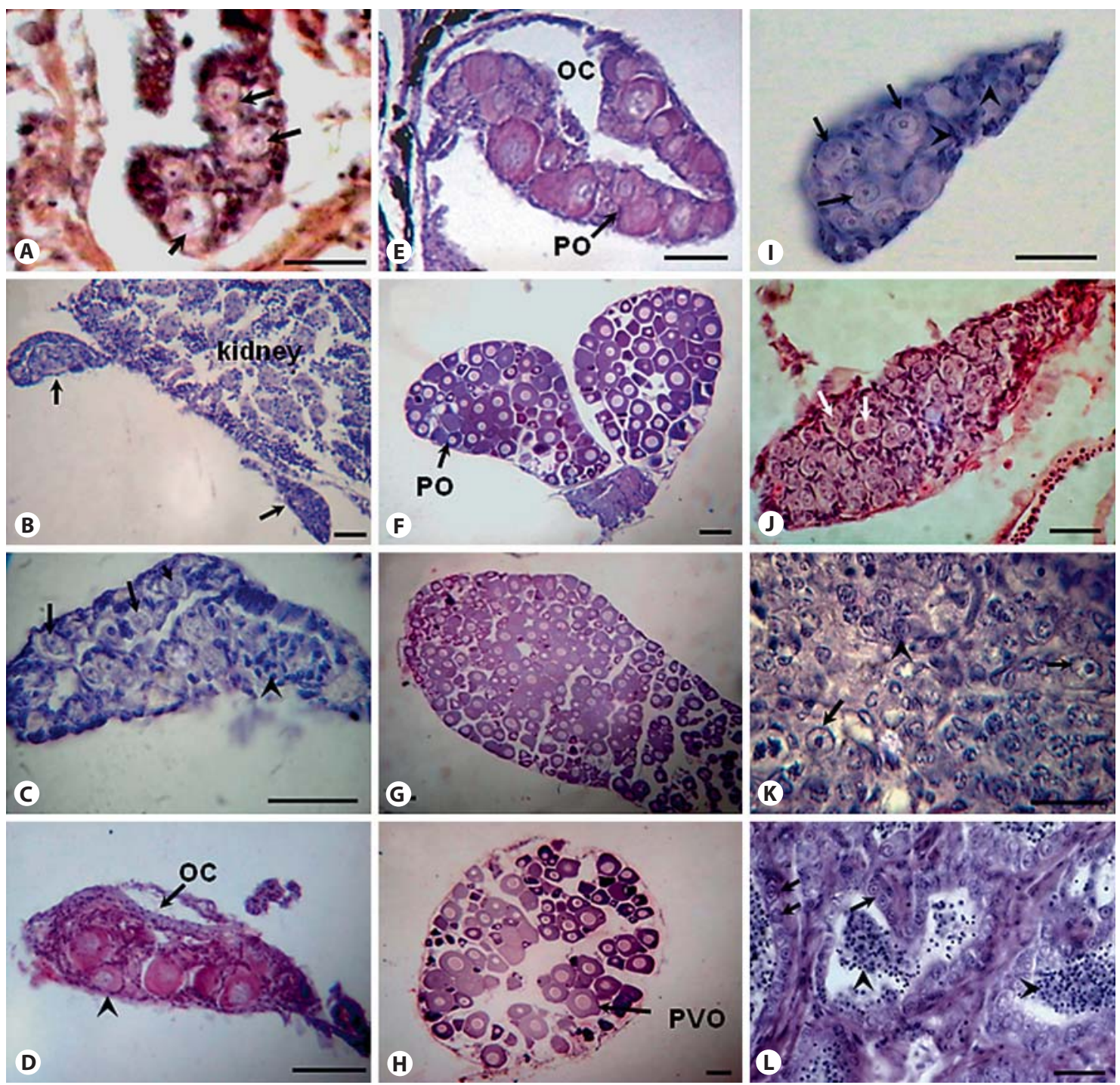

Fig. 1. Histology of gonads at different stages of development in catfish. A Primitive gonad at $28 \mathrm{dph}$ showing PGCs (arrows) surrounded by somatic/supporting cells. B Gonadal section at $40 \mathrm{dph}$ showing bipotential/indifferent gonads (arrows) attached to the mesonephros. C Section of bipotential gonad at high magnification showing germ cells (arrows) surrounded by supporting cells (arrowheads). D Differentiating female gonad at $45 \mathrm{dph}$ with perinucleolar oocytes (arrowhead). Arrow indicates the ovarian cavity. E Differentiated ovary at $52 \mathrm{dph}$ showing primary growth oocytes (arrow). F Developing ovary at $70 \mathrm{dph}$ filled with primary growth/perinucleolar oocytes (arrow). G, H 90 and 160 dph ovary, respectively, showing primary growth and pre-vitellogenic oocytes (arrow). I 52 dph differentiating testis having germ cells (arrows) surrounded by supporting cells (arrowheads). J $70 \mathrm{dph}$ developing testis showing many proliferating primary spermatogonia (arrows). K Testis section at $90 \mathrm{dph}$ showing primary spermatogonia (arrows) and differentiating spermatogonia (arrowheads). L 160 dph testis showing lumens filled with spermatids/spermatozoa (arrowheads) indicating progression of spermatogenesis. Arrows indicate primary spermatogonia. OC = Ovarian cavity; $\mathrm{PO}=$ primary growth/perinucleolar oocytes; $\mathrm{PVO}=$ pre-vitellogenic oocytes. All scale bars indicate $50 \mu \mathrm{m}$. as the development proceeds till maturity (fig. 4A-C). Cyp19a1 transcript was detectable in undifferentiated gonads at 30 and $40 \mathrm{dph}$ (fig. 4D). Thereafter, its expression was significantly higher in females than in males throughout development $\left.{ }^{* *} \mathrm{p}<0.001\right)$ (fig. $\left.4 \mathrm{D}\right) .17 \beta$-hsd1 expres- sion was noticed in both gonads after sex differentiation from 50 dph until 300 dph with abundant levels in females and very low levels in males $\left({ }^{* *} \mathrm{p}<0.001\right)$ (fig. $4 \mathrm{E}$ ). Cyp11b1 expression was not detectable in undifferentiated gonads until $40 \mathrm{dph}$ (fig. $4 \mathrm{~F}$ ). Its expression became 


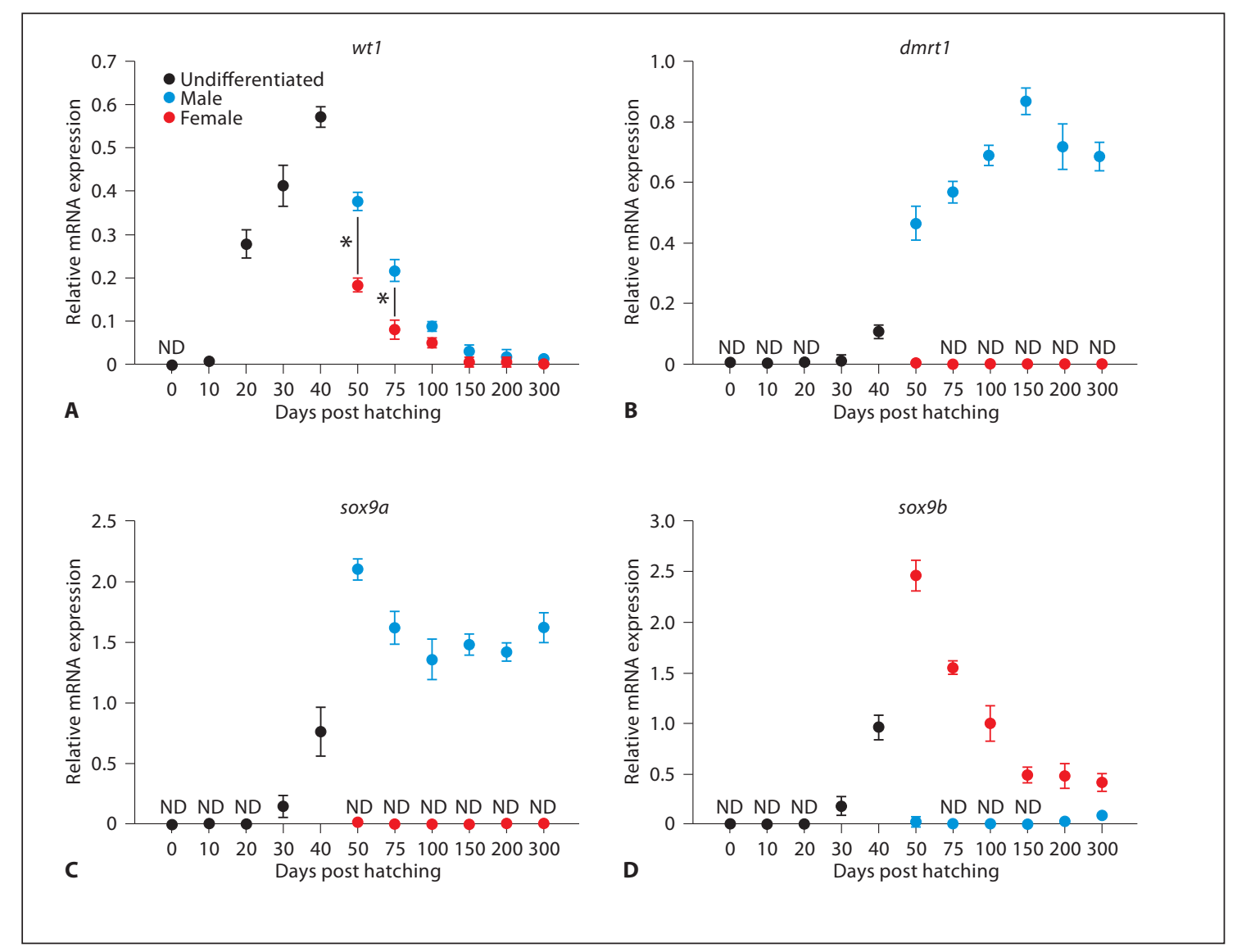

Fig. 2. Relative mRNA expression in catfish gonads from 0 to $300 \mathrm{dph}$. A wt1 (Wilms' tumor gene type 1), B $d m r t 1$ (doublesex and mab-3 related transcription factor 1), C sox $9 a$ (SRY-related homeobox gene 9 isoform a) and D sox $9 b$ (SRY-related homeobox gene 9 isoform b). ND = Non-detectable level. Statistical differences between male and female groups were determined by Student's t test $\left({ }^{*} \mathrm{p}<0.05\right)$.

apparent only in differentiating male gonads from $50 \mathrm{dph}$ to $100 \mathrm{dph}$, further increasing during the initiation of spermatogenesis in the developing testis at 150-200 dph (fig. 4F). However, barely detectable levels of cyp11b1 were noticed in female gonads around 200-300 dph when compared to male gonads ( $\mathrm{p}<0.001)$ (fig. $4 \mathrm{~F})$.

\section{IHC Localization of Dmrt1, Sox9, Cyp19a1 and Foxl2} in Differentiated Gonads

We studied the localization of Dmrt1 and Sox9 in differentiated male gonads at $52 \mathrm{dph}$ using IHC. Similarly, Foxl2 and Cyp19a1 expression was analyzed in developing female gonads. IHC studies revealed Dmrtl in both germ cells and supporting cells of male gonads at $52 \mathrm{dph}$ (fig. 5A), while Sox9 was detectable only in the supporting cells (fig. 5B). Cyp19a1 was localized in the follicular layer extending to the cytoplasm of the primary growth oocytes in the differentiated ovary at $52 \mathrm{dph}$ (fig. 5C). On the other hand, Foxl 2 was detectable in the follicular layer of the oocytes in the differentiated ovary at $60 \mathrm{dph}$ (fig. 5D).

\section{Discussion}

In the present study, we analyzed the expression profiles of genes encoding transcription factors and steroidogenic enzymes during the process of gonadal development in catfish. Parallel to this, we also tracked the structural changes in gonads during differentiation/ development using histology. Based on our histological observations in catfish, the first morphological signs of gonadal differentiation are seen around $45-50 \mathrm{dph}$, as it is evident by the formation of the ovarian cavity in the 


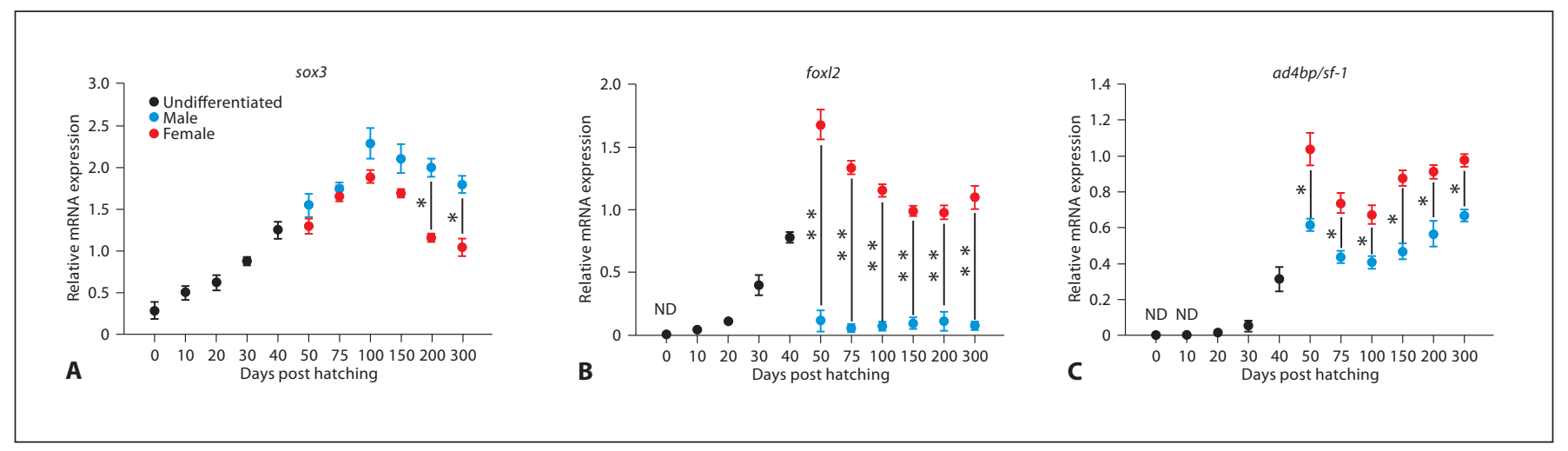

Fig. 3. Relative mRNA expression in catfish gonads from 0 to $300 \mathrm{dph}$. A sox3 (SRY-related homeobox gene 3), B foxl2 (forkhead box L2) and C ad4bp/sf-1 (steroidogenic factor-1). ND = Non-detectable level. Statistical differences between male and female groups were determined by Student's t test $\left.{ }^{*} \mathrm{p}<0.05 ;{ }^{* *} \mathrm{p}<0.001\right)$.
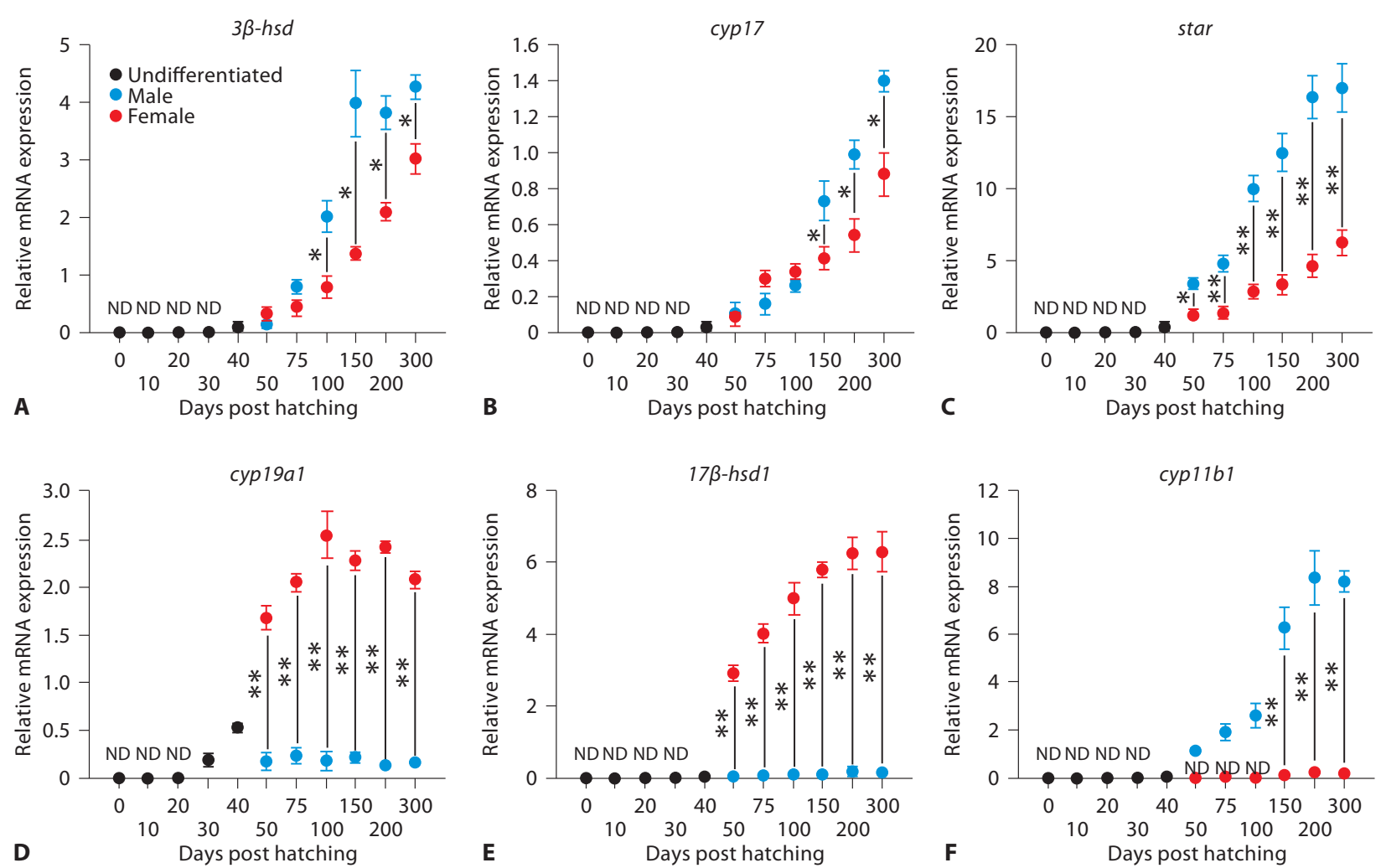

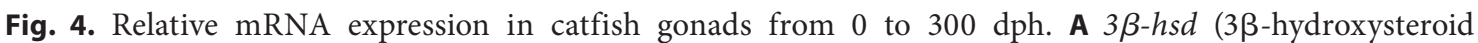
dehydrogenase/ $\Delta 4-\Delta 5$ isomerase), B cyp17 (17 $\alpha$-hydroxylase/C17-20 lyase type 1), C star (steroidogenic acute regulatory protein), D cyp19a1 (ovarian aromatase), E $17 \beta$ - $h s d 1$ (17 $\beta$-hydroxysteroid dehydrogenase type 1) and F cyp11b1 (cytochrome P450 hydroxylase type B or 11 $\beta$-hydroxylase). ND = Non detectable level. Statistical differences between male and female groups were determined by Student's t test $\left({ }^{*} \mathrm{p}<0.05 ;{ }^{* *} \mathrm{p}<0.001\right)$. 

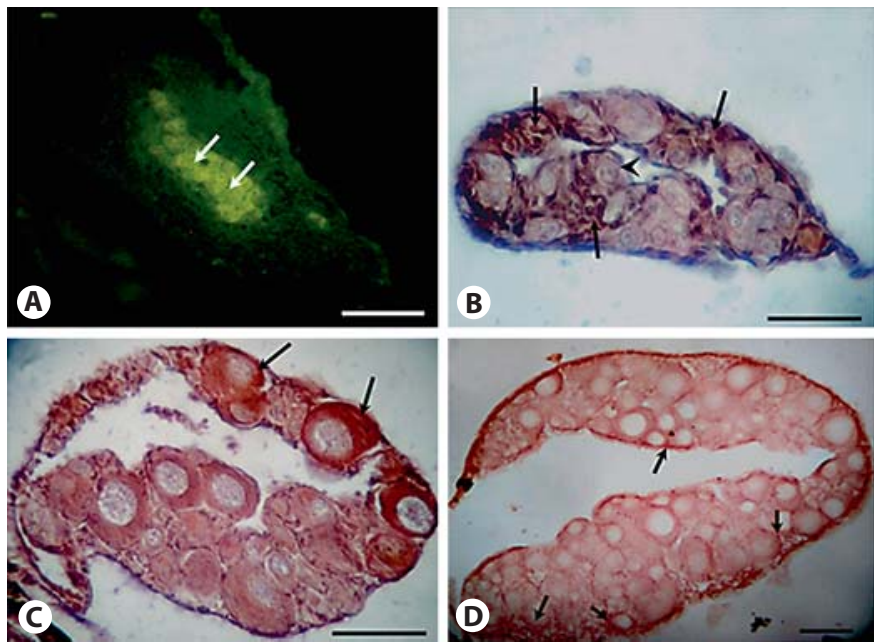

Fig. 5. Immunohistochemical localization of Dmrt1, Sox9, Cyp19a1, and Foxl2 proteins in developing gonads of catfish after FITC (A) and DAB (B-D) detection. A Dmrt1 expression (arrows) in germ cells of the male gonad at $52 \mathrm{dph}$. B Sox9 localization in the supporting (Sertoli) cells of the male gonad at $52 \mathrm{dph}$. Arrows indicate supporting cells; arrowhead indicates germ cell. c Cyp19a1 localization (arrows) was observed in the follicular layer extending inside the cytoplasm of peri-nucleolar oocytes in the female gonad at $52 \mathrm{dph}$. D Foxl2 localization in the follicular layer (arrows) of the oocytes in the female gonad at $60 \mathrm{dph}$. All scale bars indicate $50 \mu \mathrm{m}$.

differentiating female gonad. In catfish, gonads at $50 \mathrm{dph}$ are fully differentiated into either testis or ovary which was apparent by histology. In this study, we observed the beginning of ovarian differentiation much earlier than testicular differentiation as seen in the majority of gonochoristic teleosts. Interestingly, testis takes more time to differentiate fully when compared to ovary. However, a few teleosts like Mozambique tilapia and Nile tilapia show simultaneous differentiation of both sexes [Strüssmann and Nakamura, 2002].

We observed differential expression patterns of various genes/transcription factors during gonadal sex differentiation in catfish and such differences in expression become more important as the gonadal development progresses. The transcription factor WT1 is critical for the development of the urogenital system and its expression pattern is conserved in vertebrates, including teleosts [Pritchard-Jones et al., 1990; Perner et al., 2007; Klüver et al., 2009]. In catfish, wt1 expression was found very early in the indifferent gonad/MGC. Later, after differentiation of gonads, its expression was mainly seen in males and gradually decreased as the testis developed and attained maturity. The DMRT1 gene encodes a putative transcription factor that shares a highly conserved zinc finger DNA-binding domain known as DM-domain across different vertebrates [Raymond et al., 2000]. In this study, we observed dimorphic expression of $d m r t 1$ restricted to male gonads during development which is consistent with our previous results using semi-quantitative PCR where few stages of gonadal development after differentiation of gonads were analyzed [Raghuveer and Senthilkumaran, 2009]. Its expression was first evident in undifferentiated gonads and persisted throughout the development of testis till adult, suggesting an important role in testicular development in catfish. This kind of male-specific expression of dmrt1 during sex differentiation was also observed in human, tilapia, orange-spotted grouper and rainbow trout [Moniot et al., 2000; Fernandino et al., 2006; Xia et al., 2007; Kobayashi et al., 2008]. However, there are few reports where $d m r t 1$ expression was also shown in female gonads [Guo et al., 2005; Huang et al., 2005]. The IHC results revealed that Dmrtl protein was expressed in germ cells of developing testis as reported previously from our group [Raghuveer and Senthilkumaran, 2009] and also from others using teleosts like zebrafish, orange-spotted grouper and hermaphrodite grouper [Guo et al., 2005; Xia et al., 2007; Zhou and Gui, 2010]. However, in mice DMRT1 expression was seen in both germ and somatic cells [Lei et al., 2007; Matson et al., 2010]. Another testicular related gene, SOX9, that belongs to the high-mobility-group (HMG) family of transcription factors is up-regulated in male gonads during differentiation [Kent et al., 1996]. As in other teleosts, we also identified 2 isoforms of sox 9 in catfish, sox $9 a$ and sox $9 b$, which showed dimorphic expression patterns in developing catfish gonads: sox $9 a$ expression was detectable only in developing testis, while sox $9 b$ expression was mostly restricted to ovary. This kind of differential expression pattern was also reported in zebrafish and the Nile tilapia [Chiang et al., 2001; Kobayashi et al., 2008] which suggests that sox $9 a$ retained its function as testis-specific gene, while sox $9 b$ may have a new role to play in ovarian development. Sox 9 protein was localized in somatic/supporting cells in the differentiating catfish male gonad using a heterologous antibody (specific to human SOX9). In catfish female gonads, it was localized in the cytoplasm of the oocytes as reported earlier by Raghuveer and Senthikumaran [2010b]. In other teleosts, Sox9 protein was also detected in gonads of both males and females [Chiang et al., 2001; Yokoi et al., 2002; El Jamil et al., 2008]. Although its role is well defined in testis, it is not yet clear what exactly it does in ovary. The SOX 3 gene is a member 
of the HMG family of transcription factors which is expressed in gonads and brain during early vertebrate development [Weiss et al., 2003]. Moreover, Sox3 is important for normal oocyte development, testis differentiation and gametogenesis in mouse [Weiss et al., 2003]. In this study, sox3 was expressed in primordial gonads at very early stages of development as it is known to be detectable in PGCs of other teleosts [Yao et al., 2007; Zhou and Gui, 2010]. Its expression was noticed in both male and female developing gonads of catfish. Foxl2 belongs to the forkhead family of transcription factors that regulate cyp $19 a 1$ expression during ovarian differentiation in teleosts [Wang et al., 2007]. Recently, in human it has been reported that conditional knock out of FOXL2 results in the upregulation of SOX9 in the transdifferentiated ovary [Uhlenhaut et al., 2009]. In this study, foxl2 was expressed at significantly higher levels in female gonads than in male gonads during development, suggesting its role in ovarian differentiation. IHC showed Foxl2 immunoreactivity in the follicular layer of oocytes of developing female catfish. In accordance to this, in medaka and also in the Nile tilapia, Foxl2 protein was detected in the follicular layer of oocytes by IHC [Nakamoto et al., 2007; Wang et al., 2007]. Ad4bp/sf-1 was initially identified as a key regulator for steroid hormone biosynthesis [Bakke et al., 2001]. It is involved in the differentiation/development of testis and ovary by regulating the transcription of amh in males and cyp19a1 in females [Yoshiura et al., 2003; Takada et al., 2006; Wang et al., 2007]. In catfish its expression was also seen in indifferent gonads during early development. Later on, after differentiation, it was observed in both male and female gonads suggesting a role in gonadal development in both sexes.

In teleosts, sex steroids affect the development of germ cells and other cell types during the process of gonadal sex differentiation [Yamamoto, 1969; Devlin and Nagahama, 2002]. Estradiol-17 $\beta$ is considered to be responsible for inducing and maintaining ovarian development, and its levels are considerably higher in females than in males. In teleosts, testicular development is mainly regulated by the potent androgen 11-ketotestosterone (11-KT) produced by males [Nakamura et al., 1998]. In this study, we showed that $c y p 19 a 1$ which is required for the production of estradiol-17 $\beta$ was expressed very early in primordial/indifferent gonads, around 30-40 dph, prior to morphological differentiation of the gonads which was followed by a sharp increase at $50 \mathrm{dph}$ in female gonads. Later on, the levels were found to be high in ovary throughout development, which substantiates part of our earlier findings [Rasheeda et al., 2010a]. On the contrary, its expression was

Dimorphic Expression of Genes in Catfish Ontogeny very low in male gonads throughout development. Our IHC studies revealed that Cyp19al protein was detectable in the ooplasm of primary growth oocytes in addition to the follicular layer during ovarian development in catfish. Similar observations were also reported in killifish using in situ hybridization [Dong and Willett, 2008]. However, there are reports showing that Cyp19a1 protein was localized only in the follicular layer of vitellogenic oocytes [Wang et al., 2007]. Other steroidogenic enzyme genes

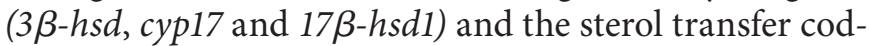
ing gene star, necessary for the synthesis of estrogens, were expressed at barely detectable levels in undifferentiated gonads at $40 \mathrm{dph}$ and thereafter increased gradually in both differentiated male and female gonads as development progressed. Similarly in the Nile tilapia most of these steroidogenic enzyme genes were also expressed in undifferentiated XX gonads (around 5-10 dph) and later gradually increased in both sexes [Ijiri et al., 2008]. Further, earlier reports on the manipulation/skewing of sex in catfish hatchlings during early development (before sex differentiation) and complete sex reversal of XY tilapia fry by following ethynylestradiol treatment prove that estrogen is produced in female gonads during the critical period of ovarian differentiation and plays a crucial role in female development [Nagahama, 2005; Raghuveer et al., 2005]. 11-KT is the most potent androgen in teleosts which is synthesized from testosterone [Nakamura et al., 1998]. Cyp11b1 encodes a key enzyme in the production of $11 \beta$ hydoxytestosterone from testosterone. An important observation in this study showed that cyp11b1 was expressed in males after gonadal differentiation (from $50 \mathrm{dph}$ onwards). On the other hand, its expression was negligible in female gonads of catfish, similar to female Nile tilapia where cyp11b1 was undetectable during ontogeny [Ijiri et al., 2008]. Previous studies on catfish $11 \beta$ - $h s d$, an important steroidogenic enzyme required for the production of $11-\mathrm{KT}$ in males, also showed a similar expression pattern as cyp11b1 [Rasheeda et al., 2010b]. These results together indicate that $11-\mathrm{KT}$ is not produced in gonads during differentiation, but is detectable only in the developing testis or at least late stages of testicular differentiation in catfish. In this study, the expression of cyp11b1 was abundant in testis around 150-200 dph, when spermatogenesis is initiated in catfish. In most teleosts, including catfish, the endogenous androgens do not appear to have an important role in testicular differentiation unlike that of estrogens during ovarian differentiation [Nakamura et al., 1998; Ijiri et al., 2008; present study]. Taken together, these results suggest that steroidogenic enzyme genes play a significant role in the maintenance of post-differentiation 
gonadogenesis. However, their role in ovarian differentiation, consistent with earlier reports, cannot be ruled out.

In conclusion, male-specific expression of dmrt1, sox $9 a$ and $w t 1$ in developing gonads indicates that these genes are essential for testicular differentiation. In contrast, abundant expression of sox $9 b$, foxl2 and cyp19a1 in female gonads suggests an important role for these correlates in ovarian differentiation. Results from IHC for various gene products support this contention to some extent. Thus, most of these transcription factors/genes were expressed in gonads during 30-40 dph, a period critical for the differentiation of indifferent gonads into either testis or ovary in catfish.

\section{Acknowledgements}

A Grant-in-Aid from the University Grants Commission (F. No. 34-412/2008) awarded to B.S. and a DST-PURSE grant from the University of Hyderabad (UH), India supported this work. K.R., P.S., R.S. and R.M. thank the Council of Scientific and Industrial Research/University Grant Commission for junior/senior research fellowship. We thank our Vice-Chancellor, Prof. Seyed E. Hasnain, and the Dean, Prof. M. Ramanadham, for allowing us to use the Genomics and Microarray facility of the School of Life Sciences, UH, partially sponsored by DBT-CREBB, India. All authors thank Prof. Yoshitaka Nagahama from the National Institute of Basic Biology, Okazaki, Japan for his generous gift of Cyp19a1 antiserum for our research.

\section{References}

Bakke M, Zhao L, Hanley NA, Parker KL: SF-1: a critical mediator of steroidogenesis. Mol Cell Endocrinol 171:5-7 (2001).

-Chiang EF, Pai CI, Wyatt M, Yan YL, Postlethwait J, Chung B: Two sox9 genes on duplicated zebrafish chromosomes: expression of similar transcription activators in distinct sites. Dev Biol 231:149-163 (2001).

Devlin RH, Nagahama Y: Sex determination and sex differentiation in fish: an overview of genetic, physiological, and environmental influences. Aquaculture 208:191-364 (2002).

Dong W, Willett KL: Local expression of CYP19A1 and CYP19A2 in developing and adult killifish (Fundulus heteroclitus). Gen Comp Endocrinol 155:307-317 (2008).

-El Jamil A, Kanhoush R, Magre S, Boizet-Bonhoure B, Penrad-Mobayed M: Sex-specific expression of SOX9 during gonadogenesis in the amphibian Xenopus tropicalis. Dev Dyn 237:2996-3005 (2008).

-Fernandino JI, Guilgur LG, Somoza GM: Dmrt1 expression analysis during spermatogenesis in pejerrey, Odontesthes bonariensis. Fish Physiol Biochem 32:231-240 (2006).

Guerrero-Estévez S, Moreno-Mendoza N: Sexual determination and differentiation in teleost fish. Rev Fish Biol Fish 20:101-121 (2010).

Guo Y, Cheng H, Huang X, Gao S, Yu H, Zhou R: Gene structure, multiple alternative splicing, and expression in gonads of zebrafish Dmrt1. Biochem Biophys Res Commun 330: 950-957 (2005).

-Huang X, Guo Y, Shui Y, Gao S, Yu H, et al: Multiple alternative splicing and differential expression of dmrt1 during gonad transformation of the rice field eel. Biol Reprod 73:10171024 (2005).

Hughes IA: Minireview: sex differentiation. Endocrinology 142: 3281-3287 (2001).
Ijiri S, Kaneko H, Kobayashi T, Wang DS, Sakai F, et al: Sexual dimorphic expression of genes in gonads during early differentiation of a teleost fish, the Nile tilapia Oreochromis niloticus. Biol Reprod 78:333-341 (2008).

Kent J, Wheatley SC, Andrews JE, Sinclair AH, Koopman P: A male-specific role for SOX9 in vertebrate sex determination. Development 122:2813-2822 (1996).

Klüver N, Herpin A, Braasch I, Driessle J, Schartl M: Regulatory back-up circuit of medaka Wt1 co-orthologs ensures PGC maintenance. Dev Biol 325:179-188 (2009).

-Kobayashi T, Kajiura-Kobayashi H, Guan G, Nagahama Y: Sexual dimorphic expression of $D M R T 1$ and Sox9a during gonadal differentiation and hormone-induced sex reversal in the teleost fish Nile tilapia (Oreochromis niloticus). Dev Dyn 237:297-306 (2008).

Kondo M, Nanda I, Schmid M, Schartl M: Sex determination and sex chromosome evolution: insights from medaka. Sex Dev 3:88-98 (2009).

Lei N, Hornbaker KI, Rice DA, Karpova T, Agbor VA, Heckert LL: Sex-specific differences in mouse DMRT1 expression are both cell type- and stage-dependent during gonad development. Biol Reprod 77:466-475 (2007).

Matson CK, Murphy MW, Griswold MD, Yoshida S, Bardwell VJ, Zarkower D: The mammalian doublesex homolog DMRT1 is a transcriptional gatekeeper that controls the mitosis versus meiosis decision in male germ cells. Dev Cell 19:612-624 (2010).

Matsuda M, Nagahama Y, Shinomiya A, Sato T, Matsuda C, et al: DMY is a Y-specific DMdomain gene required for male development in the medaka fish. Nature 417:559-563 (2002).

-Moniot B, Berta P, Scherer G, Südbeck P, Poulat F: Male specific expression suggests role of $D M R T 1$ in human sex determination. Mech Dev 91:323-325 (2000).
Nagahama Y: Molecular mechanisms of sex determination and gonadal sex differentiation in fish. Fish Physiol Biochem 31:105-109 (2005).

Nakamoto M, Wang DS, Suzuki A, Matsuda M, Nagahama Y, Shibata N: Dax1 suppresses P450arom expression in medaka ovarian follicles. Mol Reprod Dev 74:1239-1246 (2007).

- Nakamura M, Kobayashi T, Chang X-T, Nagahama Y: Gonadal sex differentiation in teleost fish. J Exp Zool 281:362-372 (1998).

-Nanda I, Kondo M, Hornung U, Asakawa S, Winkler C, et al: A duplicated copy of $D M R T 1$ in the sex-determining region of the Y chromosome of the medaka, Oryzias latipes. Proc Natl Acad Sci USA 99:1177811783 (2002).

Perner B, Englert C, Bollig F: The Wilms tumor genes wt1a and $w t 1 b$ control different steps during formation of the zebrafish pronephros. Dev Biol 309:87-96 (2007).

Pritchard-Jones K, Fleming S, Davidson D, Bickmore W, Porteous D, et al: The candidate Wilms' tumor gene is involved in genitourinary development. Nature 346:194-197 (1990).

Raghuveer K, Senthilkumaran B: Identification of multiple dmrt1s in catfish: localization, dimorphic expression pattern, changes during testicular cycle and after methyltestosterone treatment. J Mol Endocrinol 42:437448 (2009).

Raghuveer K, Senthilkumaran B: Cloning and differential expression pattern of vasa in the developing and recrudescing gonads of catfish, Clarias gariepinus. Comp Biochem Physiol A Mol Integr Physiol 157:79-85 (2010a).

Raghuveer K, Senthilkumaran B: Isolation of sox9 duplicates in catfish: localization, differential expression pattern during gonadal development and recrudescence, and hCGinduced up-regulation of $\operatorname{sox} 9$ in testicular slices. Reproduction 140:477-487 (2010b). 
Raghuveer K, Garhwal R, Wang DS, Bogerd J, Kirubagaran R, et al: Effect of methyl testosterone- and ethynyl estradiol-induced sex differentiation on catfish, Clarias gariepinus: expression profiles of DMRT1, cytochrome P450aromatases and $3 \beta$ hydroxysteroid dehydrogenase. Fish Physiol Biochem 31:143-147 (2005).

Rasheeda MK, Sridevi P, Senthilkumaran B: Cytochrome P450 aromatases: impact on gonadal development, recrudescence and effect of hCG in the catfish, Clarias gariepinus. Gen Comp Endocrinol 167:234-245 (2010a).

Rasheeda MK, Kagawa H, Kirubagaran R, Dutta-Gupta A, Senthilkumaran B: Cloning, expression and enzyme activity analysis of testicular 11ß-hydroxysteroid dehydrogenase during seasonal cycle and after hCG induction in air-breathing catfish Clarias gariepinus. J Steroid Biochem Mol Biol 120:1-10 (2010b).

Raymond CS, Murphy MW, O'Sullivan MG, Bardwell VJ, Zarkower D: Dmrt1, a gene related to worm and fly sexual regulators, is required for mammalian testis differentiation. Genes Dev 14:2587-2595 (2000).

\section{$\checkmark$}

rüssmann CA, Nakamura M: Morphology, endocrinology, and environmental modulation of gonadal sex differentiation in teleost fishes. Fish Physiol Biochem 26:13-29 (2002).

Takada S, Wada T, Kaneda R, Choi YL, Yamashita Y, Mano H: Evidence for activation of Amh gene expression by steroidogenic factor 1 . Mech Dev 123:472-480 (2006).

Uhlenhaut NH, Jakob S, Anlag K, Eisenberger T, Sekido R, et al: Somatic sex reprogramming of adult ovaries to testes by FOXL2 ablation. Cell 139:1130-1142 (2009).

Wang DS, Kobayashi T, Zhou LY, Paul-Prasanth B, Ijiri S, et al: Foxl2 up-regulates aromatase gene transcription in a female-specific manner by binding to the promoter as well as interacting with ad4 binding protein/steroidogenic factor 1. Mol Endocrinol 21:712-725 (2007).

Weiss J, Meeks JJ, Hurley L, Raverot G, Frassetto A, Jameson JL: Sox3 is required for gonadal function, but not sex determination, in males and females. Mol Cell Biol 23:80848091 (2003).

Xia W, Zhou L, Yao B, Li C-J, Gui J-F: Differential and spermatogenic cell-specific expression of DMRT1 during sex reversal in protogynous hermaphroditic groupers. Mol Cell Endocrinol 263:156-172 (2007).
Yamamoto T: Sex differentiation, in Hoar WS, Randall DJ (eds): Fish Physiology, Vol. III. Reproduction and Growth. Bioluminescence, Pigments, and Poisons, pp 117-175 (Academic Press, New York 1969).

Yao B, Zhou L, Wang Y, Xia W, Gui JF: Differential expression and dynamic changes of SOX3 during gametogenesis and sex reversal in protogynous hermaphroditic fish. J Exp Zool A Ecol Genet Physiol 307:207-219 (2007).

Yokoi H, Kobayashi T, Tanaka M, Nagahama Y, Wakamatsu Y, et al: Sox9 in a teleost fish, medaka (Oryzias latipes): evidence for diversified function of Sox9 in gonad differentiation. Mol Reprod Dev 63:5-16 (2002).

-Yoshiura Y, Senthilkumaran B, Watanabe M, Oba Y, Kobayashi T, Nagahama Y: Synergistic expression of Ad4BP/SF-1 and cytochrome P-450 aromatase (ovarian type) in the ovary of Nile tilapia, Oreochromis niloticus, during vitellogenesis suggests transcriptional interaction. Biol Reprod 68:1545-1553 (2003).

Zhou L, Gui J-F: Molecular mechanisms underlying sex change in hermaphroditic groupers. Fish Physiol Biochem 36:181-193 (2010). 